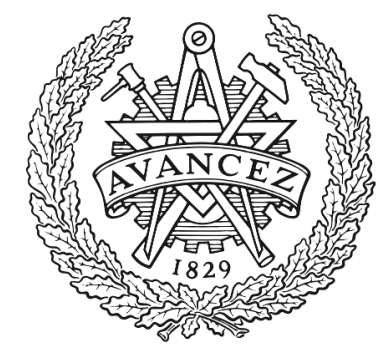

CHALMERS

UNIVERSITY OF TECHNOLOGY

\title{
Construction of a universal twist element from an R-matrix
}

Downloaded from: https://research.chalmers.se, 2023-04-26 11:49 UTC

Citation for the original published paper (version of record):

Stolin, A., Kulish, P., Damaskinsky, E. (2005). Construction of a universal twist element from an

R-matrix. Journal of Mathematical Sciences, 125(2): 229-238.

http://dx.doi.org/10.1023/B:JOTH.0000049574.67818.ed

N.B. When citing this work, cite the original published paper. 


\title{
CONSTRUCTION OF A UNIVERSAL TWIST ELEMENT FROM AN $R$-MATRIX
}

\author{
A. A. Stolin, P. P. Kulish, and E. V. Damaskinsky
}

UDC 157.9

We propose a method for construction of a universal twist element based on a constant quasi-classical unifary matrix solution of the Yang-Baxter equation. The method is applied to few known R-matrices corresponding to Lie (super) algebras of rank one. Bibliography: 13 titles.

\section{INTRODUCTION}

Quantum groups were introduced by Drinfel'd [1] as an important class of Hopf algebras. In the theory of quantum groups, the universal $R$-matrix is an essential object intertwining the coproduct $\Delta$ with the opposite coproduct $\Delta^{\mathrm{op}}$ by the following equality:

$$
\mathcal{R} \Delta=\Delta^{\mathrm{op}} \mathcal{R} .
$$

Another important object, introduced later, is a twist transformation (twist) [2,3],

$$
\Delta \rightarrow \Delta_{t}=\mathcal{F} \Delta \mathcal{F}^{-1}
$$

In the formulas above, $\mathcal{R}$ and $\mathcal{F}$ are elements of the tensor square of the Hopf algebra. Twist elements are known explicitly for some quantum groups, in particular, for quantum deformations of the universal enveloping algebras of some Lie algebras.

The FRT-formalism [4] allows us to reconstruct the quantum group corresponding to some known matrix representation of the universal $R$-matrix. However, this method does not give us explicit expressions for universal elements.

A similar result for universal twists was obtained by Drinfel'd [2]. It was shown that if we know a unitary matrix $R$ that is a quasiclassical solution of the Yang-Baxter equation, then there exists a universal twist $\mathcal{F}$ such that $\mathcal{R}=\mathcal{F}_{21} \mathcal{F}^{-1}$ in the vector representation. This situation is similar to that mentioned above: if a matrix solution of the Yang-Baxter equation is known, then a formal reconstruction of the universal element is possible. However, as in the previous case, there is no computational method to obtain explicit expressions for the universal twist $\mathcal{F}$. Construction of universal twist elements is a rather complicated problem. Some explicit expressions can be found in $[5,6]$ (see also references therein).

In the present paper, we suggest a method of constructing a universal twist element for a given unitary quasiclassical matrix solution of the Yang-Baxter equation. This method is based on investigation of the associative algebra defined by the matrix relation $R T_{1} T_{2}=T_{2} T_{1}$, where $T=\left\{t_{i j} \in U\left(g l_{n}\right)^{*}\right\}$. This algebra is a deformation of the algebra of polynomial functions on the group $G L_{n}$ (in some examples, it is possible to consider its subgroups). The above-mentioned associative algebra is isomorphic, as a left $U\left(g \ell_{n}\right)$-module algebra, to the nondeformed (commutative) algebra Fun of polynomials on $G L_{n}$ (certainly, this isomorphism is not an algebra isomorphism). Let us recall that there exists a nondegenerate pairing between the algebras Fun and $U\left(g \ell_{n}\right)$. Hence, there is a nondegenerate pairing between $U\left(g \ell_{n}\right)$ and the algebra generated by the relation $R T_{1} T_{2}=T_{2} T_{1}$. This duality generates a coassociative operation $d: U\left(g \ell_{n}\right) \rightarrow U\left(g \ell_{n}\right) \otimes U\left(g \ell_{n}\right)$ (which is not an algebra homomorphism). In this case, $\mathcal{F}=d(1)$ is the required twist.

This twist $\mathcal{F} \in \mathcal{H} \otimes \mathcal{H}$ allows us to introduce an additional coalgebra structure in the Hopf algebra $\mathcal{H}$ as follows:

$$
d: \mathcal{H} \rightarrow \mathcal{H} \otimes \mathcal{H}, \quad d:=\mathcal{F} \Delta .
$$

Structures of that type were considered in the works $[7,8]$ devoted to analysis of the Lie-Poisson groups.

In Sec. 2, we discuss a general connection between the universal twist $\mathcal{F}$ of a Hopf algebra $\mathcal{H}$ and the matrix form of the $R T T$-relation; this connection defines a new multiplication law on the dual Hopf algebra $\mathcal{H}^{*}$. In Sec. 3, we describe some examples of application of this connection for reconstruction of the universal twisting element from a given $R$-matrix in the case of Lie (super)algebras of rank 1. Some details of the calculations are given in the Appendix.

Translated from Zapiski Nauchnykh Seminarov POMI, Vol. 291, 2003, pp. 228-244. Original article submitted September 15, 2002. 


\section{Module Algebras AND TWists}

Let us consider in a given Hopf algebra $\mathcal{H}$ an element $\mathcal{F} \in \mathcal{H} \otimes \mathcal{H}$ satisfying the following twist equation (the 2-cocycle condition):

$$
\mathcal{F}_{12}(\Delta \otimes i d) \mathcal{F}=\mathcal{F}_{23}(i d \otimes \Delta) \mathcal{F}
$$

and the relation

$$
(\varepsilon \otimes i d) \mathcal{F}=(i d \otimes \varepsilon) \mathcal{F}=1 \otimes 1 .
$$

Such an element $\mathcal{F}$ is called the universal twist or universal twist element.

Define a map $d_{\mathcal{F}}: \mathcal{H} \rightarrow \mathcal{H} \otimes \mathcal{H}$ by $d_{\mathcal{F}}(a)=\mathcal{F} \Delta(a)$. It is easy to show that the map $d_{\mathcal{F}}$ satisfies the following conditions:

$$
(d \otimes i d) d=(i d \otimes d) d \quad(\text { coassociativity })
$$

and

$$
d_{\mathcal{F}}(a b)=d_{\mathcal{F}}(a) \Delta(b)
$$

Lemma 2.1. For a twist $\mathcal{F}$, the following conditions hold:

(a) $d_{\mathcal{F}}$ is a coassociative map;

(b) $d_{\mathcal{F}}(a b)=d_{\mathcal{F}}(a) \Delta(b)$;

(c) $(\varepsilon \otimes i d) d_{\mathcal{F}}(a)=1 \otimes a \quad$ and $\quad(i d \otimes \varepsilon) d_{\mathcal{F}}(a)=a \otimes 1$.

Conversely, if $d: \mathcal{H} \rightarrow \mathcal{H} \otimes \mathcal{H}$ satisfies conditions (a), (b), and (c), then $\mathcal{F}=d(1)$ is a twist.

Proof. We have already shown that a twist $\mathcal{F}$ generates the map $d_{\mathcal{F}}(a)=\mathcal{F} \Delta(a)$ satisfying conditions (a) and (b), while condition (c) is satisfied automatically.

Conversely, if a map $d: \mathcal{H} \rightarrow \mathcal{H} \otimes \mathcal{H}$ is given, then properties (a) and (b) imply that the map

$$
\mathcal{F}=d(1):=\sum f_{1} \otimes f_{2}
$$

satisfies the relation

$$
\mathcal{F}_{12}(\Delta \otimes i d)(\mathcal{F})=\mathcal{F}_{23}(i d \otimes \Delta)(\mathcal{F}) .
$$

Putting $a=1$ in $(\mathrm{c})$, we see that $(\varepsilon \otimes i d) \mathcal{F}=(i d \otimes \varepsilon) \mathcal{F}=1 \otimes 1$.

Let us consider the vector space $\mathcal{H}^{*}$ dual to $\mathcal{H}$. We note that $\mathcal{H}^{*}$ is a right $\mathcal{H}_{o p}$-module since

$$
\langle\alpha, a b\rangle=\left\langle\alpha_{(1)}, a\right\rangle\left\langle\alpha_{(2)}, b\right\rangle:=\langle\alpha \tilde{b}, a\rangle, \quad \text { where } \quad \alpha \tilde{b}=\alpha_{(1)}\left\langle\alpha_{(2)}, b\right\rangle
$$

$\alpha \in \mathcal{H}^{*}, a, b \in \mathcal{H}$, and $\mathcal{H}_{o p}$ is the Hopf algebra $\mathcal{H}$ with the opposite multiplication. A map $d: \mathcal{H} \rightarrow \mathcal{H} \otimes \mathcal{H}$ satisfying conditions (a)-(c) induces an associative multiplication law on $\mathcal{H}^{*}$ as follows:

$$
\begin{gathered}
\langle\alpha \circ \beta, a\rangle=\langle\alpha \otimes \beta, d(a)\rangle=\left\langle\alpha \otimes \beta, f_{1} a_{(1)} \otimes f_{2} a_{(2)}\right\rangle \\
=\left\langle\alpha, f_{1} a_{1}\right\rangle\left\langle\beta, f_{2} a_{(2)}\right\rangle=\left\langle\Delta^{*}(\alpha), f_{1} \otimes a_{(1)}\right\rangle\left\langle\Delta^{*}(\beta), f_{2} \otimes a_{(2)}\right\rangle \\
=\left\langle\alpha_{(1)}, f_{1}\right\rangle\left\langle\alpha_{(2)}, a_{(1)}\right\rangle\left\langle\beta_{(1)}, f_{2}\right\rangle\left\langle\beta_{(2)}, a_{(2)}\right\rangle \\
=\left\langle\alpha_{(1)}, f_{1}\right\rangle\left\langle\beta_{(1)}, f_{2}\right\rangle\left\langle\alpha_{(2)}, a_{(1)}\right\rangle\left\langle\beta_{(2)}, a_{(2)}\right\rangle \\
=\left\langle\alpha_{(1)}, f_{1}\right\rangle\left\langle\beta_{(1)}, f_{2}\right\rangle\left\langle\alpha_{(2)} \otimes \beta_{(2)}, a_{(1)} \otimes a_{(2)}\right\rangle \\
=\left\langle\alpha_{(1)}, f_{1}\right\rangle\left\langle\beta_{(1)}, f_{2}\right\rangle\left\langle\alpha_{(2)} \otimes \beta_{(2)}, \Delta(a)\right\rangle=\left\langle\alpha_{(1)} \otimes \beta_{(1)}, \mathcal{F}\right\rangle\left\langle\alpha_{(2)} \otimes \beta_{(2)}, \Delta(a)\right\rangle \\
\left.=\beta_{(1)} \otimes \alpha_{(2)} \otimes \beta_{(2)}, \mathcal{F} \otimes \Delta(a)\right\rangle=\left\langle\sigma_{23}\left(\alpha_{(1)} \otimes \alpha_{(2)} \otimes \beta_{(1)} \otimes \beta_{(2)}\right), \mathcal{F} \otimes \Delta(a)\right\rangle \\
=\left\langle\sigma_{23} \Delta^{*}(\alpha) \otimes \Delta^{*}(\beta), \mathcal{F} \otimes \Delta(a)\right\rangle .
\end{gathered}
$$

Hence, $\alpha \circ \beta=\left\langle\alpha_{(1)} \otimes \beta_{(1)}, \mathcal{F}\right\rangle \alpha_{(2)} \beta_{(2)}$.

Proposition 2.2. Twist elements are in a one-to-one correspondence with module algebra structures on $\mathcal{H}^{*}$ (over $\mathcal{H}_{o p}$ ) such that $\varepsilon \circ f=f \circ \varepsilon=f$. 
Corollary 2.3. Let $\left\{e_{0}=1, e_{1}, e_{2}, \ldots\right\}$ and $\left\{e^{0}=\varepsilon, e^{1}, e^{2}, \ldots\right\}$ be dual bases in $\mathcal{H}$ and $\mathcal{H}^{*}$, respectively, and let

$$
e^{i} \circ e^{j}=\sum_{k} m_{k}^{i j} e^{k}
$$

Then

$$
d(1)=\sum_{r, s} m_{0}^{r s} e_{r} \otimes e_{s} \quad \text { and } \quad m_{r}^{0 k}=m_{r}^{k 0}=\delta_{r}^{k}
$$

Indeed,

$$
\begin{aligned}
\left\langle e^{j} \circ e^{i}, e_{p}\right\rangle & =\left\langle e^{j} \otimes e^{i}, d\left(e_{p}\right)\right\rangle, \\
\left\langle e^{j} \circ e^{i}, e_{p}\right\rangle & =\left\langle\sum_{k} \stackrel{\circ}{m}_{k}^{j i} e^{k}, e_{p}\right\rangle=\sum_{k} \stackrel{\circ}{m}_{k}^{j i}\left\langle e^{k}, e_{p}\right\rangle=\stackrel{\circ}{m}_{p}^{j i}, \\
\text { and } & \\
\left\langle e^{j} \otimes e^{i}, d\left(e_{p}\right)\right\rangle & =\left\langle e^{j} \otimes e^{i}, \sum_{r, s} d_{p}^{r s} e_{r} \otimes e_{s}\right\rangle=\sum_{r, s} d_{p}^{r s}\left\langle e^{j} \otimes e^{i}, e_{r} \otimes e_{s}\right\rangle \\
& =\sum_{r, s} d_{p}^{r s}\left\langle e^{j}, e_{r}\right\rangle\left\langle e^{i}, e_{s}\right\rangle=d_{p}^{j i} .
\end{aligned}
$$

Thus, $d_{p}^{j i}=\stackrel{\circ}{m_{p}^{j i}}$

$$
d\left(e_{p}\right):=\sum_{r, s} d_{p}^{r s} e_{r} \otimes e_{s}=\sum_{r, s} \stackrel{\circ}{m_{p}^{r s}} e_{r} \otimes e_{s}
$$

and

$$
d\left(e_{p}\right)=d\left(1 e_{p}\right)=d(1) \Delta\left(e_{p}\right)=\mathcal{F} \Delta\left(e_{p}\right) .
$$

\section{EXAMPLES}

The case of $U\left(b_{2}\right)$

Let $\mathcal{H}=U\left(b_{2}\right)$ be the universal enveloping algebra of a Borel algebra with generators $h$ and $x$ and the defining relation $[h, x]=x$. It is known that the monomials $e_{m, k}=h^{m} x^{k}, m, k=0,1,2, \ldots$, form a linear basis in $\mathcal{H}$ (the PBW-theorem). Let us consider the Jordanian $R$-matrix,

$$
R=\left(\begin{array}{cccc}
1 & -\xi & \xi & \xi^{2} \\
0 & 1 & 0 & -\xi \\
0 & 0 & 1 & \xi \\
0 & 0 & 0 & 1
\end{array}\right)
$$

which is a unitary solution of the Yang-Baxter equation [9] (i.e., $R_{21} R=1 \otimes 1$ ) and quasiclassical. According to [2], there exists a twist $\mathcal{F} \in \mathcal{H} \otimes \mathcal{H}$ such that $R=(\rho \otimes \rho)\left(\mathcal{F}_{21} \mathcal{F}^{-1}\right)$, where $\rho$ is the vector representation of $U\left(b_{2}\right)$. To find $\mathcal{F}$, we consider the following algebra (which is not a Hopf algebra):

$$
\mathcal{A}=\frac{\mathbb{C}\left\langle t_{11}, t_{12}\right\rangle}{R T_{1} \cdot T_{2}=T_{2} \cdot T_{1}} .
$$

Here

$$
T_{1}=T \otimes 1=\left(\begin{array}{cccc}
t_{11} & 0 & t_{12} & 0 \\
0 & t_{11} & 0 & t_{12} \\
0 & 0 & t_{11}^{-1} & 0 \\
0 & 0 & 0 & t_{11}^{-1}
\end{array}\right)
$$

and

$$
T_{2}=1 \otimes T=\left(\begin{array}{cccc}
t_{11} & t_{12} & 0 & 0 \\
0 & t_{11^{-1}} & 0 & 0 \\
0 & 0 & t_{11} & t_{12} \\
0 & 0 & 0 & t_{11}^{-1}
\end{array}\right)
$$

Let $\mathbb{C}\left\langle t_{11}, t_{12}\right\rangle$ be the algebra generated by the noncommutative elements $t_{11}$ and $t_{12}$ satisfying the relation $R T_{1} \cdot T_{2}=T_{2} \cdot T_{1}$. 
Proposition 3.1. (1) $\mathcal{A}=\frac{\mathbb{C}\left\langle t_{11}, t_{12}\right\rangle}{\left[t_{11}, t_{12}\right]=\xi \cdot 1}$.

(2) $\mathcal{A}$ is a left module algebra over $\mathcal{H}=U\left(b_{2}\right)$ with the following action:

$$
\tilde{x} t_{12}=t_{11}, \tilde{h} t_{12}=-\frac{1}{2} t_{12}, \tilde{x} t_{11}=0, \tilde{h} t_{11}=\frac{1}{2} t_{11} .
$$

Proof. Both statements can be checked immediately. It is also possible to prove these statements as follows. First of all, it is easy to prove the Poincare-Birkhoff-Witt theorem: the monomials

$$
f^{k, m}=t_{11}^{k} t_{12}^{n}
$$

form a linear basis in $\mathcal{A}$. Let us consider the algebra of polynomials on the group $B_{2}$,

$$
\text { Fun }=\frac{\mathbb{C}\left\langle t_{11}, t_{12}\right\rangle}{T_{1} T_{2}=T_{2} T_{1}}=\mathbb{C}\left[t_{11}, t_{12}\right] \text {. }
$$

It is known that $F u n$ is both a left $U\left(b_{2}\right)$-module algebra and a right $U\left(b_{2}\right)_{o p}$-module algebra. It is also known that $U\left(b_{2}\right)$ acts on Fun by left derivatives, while $U\left(b_{2}\right)_{o p}$ acts by right derivatives. Since $R$ stands on the left in the defining relation, it is possible to show that the structure of the left $U\left(b_{2}\right)$-module algebra on $\mathcal{A}$ is inherited from Fun.

Corollary 3.2. The algebras $\mathcal{A}$ and Fun are isomorphic as left $U\left(b_{2}\right)$-module algebras.

Let us recall that the used pairing between $U\left(b_{2}\right)$ and $F u n$ is determined by the relation $\langle f, b\rangle=f \tilde{b}(e)$, where $f \in F u n, b \in U\left(b_{2}\right)$, and $e$ is the unit element of the group $B_{2}$.

Thus, we have to find dual bases in $U\left(b_{2}\right)$ and Fun. However, it is known that this is impossible. Therefore, we have to extend the algebra Fun. We construct such an extension by the method suggested in [10]. We recall that Fun is a Hopf algebra and the above-mentioned dualization is a pairing of the Hopf algebras Fun and $U\left(b_{2}\right)_{o p}$.

Let $\varphi$ and $\omega$ denote the elements dual to $h$ and $x$, respectively. Then

$$
\left\langle h^{k} x^{m}, \varphi^{p} \omega^{q}\right\rangle=k ! m ! \cdot \delta_{k, p} \cdot \delta_{m, q},
$$

and the tensor

$$
\mathcal{T}=\exp (h \otimes \varphi) \exp (x \otimes \omega) \in U\left(b_{2}\right) \otimes \widetilde{F u n}
$$

is a canonical element. We have denoted by $\widetilde{\text { Fun }}$ the Hopf algebra generated by the elements $\varphi, \omega$, and $e^{ \pm \varphi / 2}$, where $\Delta \varphi$ and $\Delta \omega$ are defined by

$$
\left\langle\Delta(a), h^{k} x^{m} \otimes h^{p} x^{q}\right\rangle=\left\langle a, h^{k} x^{m} h^{p} x^{q}\right\rangle .
$$

It is possible to check that $\varphi$ is a primitive element and $\Delta \omega=\omega \otimes e^{-\varphi}+1 \otimes \omega$. Thus, it is clear that $\widetilde{\text { Fun }}$ is a right module algebra over $U\left(b_{2}\right)_{o p}$.

If $\mathcal{F} \in U\left(b_{2}\right) \otimes U\left(b_{2}\right)$ is a twist, then we define a new multiplication on $\widetilde{\text { Fun }}$. Since $\mathcal{F}$ defines a coassociative operation $d_{\mathcal{F}}(a)=\mathcal{F} \Delta(a)$ on $U\left(b_{2}\right)$, then by virtue of the duality between $U\left(b_{2}\right)$ and $\widetilde{F u n}$, we obtain an associative multiplication on $\widetilde{F u n}$ which can be described as follows.

Let us define

and also

$$
\mathcal{T}_{1}=\exp (h \otimes 1 \otimes \varphi) \exp (x \otimes 1 \otimes \omega), \quad \mathcal{T}_{2}=1 \otimes \mathcal{T},
$$

$$
\widetilde{T}_{1}=(\rho \otimes \rho \otimes i d) \mathcal{T}_{1} \quad \text { and } \quad \widetilde{T}_{2}=(\rho \otimes \rho \otimes i d) \mathcal{T}_{2},
$$

where $\rho$ denotes the 2 -dimensional vector representation of the algebra $U\left(b_{2}\right)$. In this case, $\mathcal{T}_{1} \mathcal{T}_{2}=\mathcal{T}_{2} \mathcal{T}_{1}$. Further, we define $\mathcal{T}_{1} \circ \mathcal{T}_{2}$ by

Then it is possible to prove that

$$
\mathcal{T}_{1} \circ \mathcal{T}_{2}=\mathcal{F}_{12}(\Delta \otimes i d)(\mathcal{T}) .
$$

$$
\mathcal{T}_{2} \circ \mathcal{T}_{1}=\mathcal{F}_{21}\left(\Delta_{o p} \otimes i d\right)(\mathcal{T}) .
$$

Indeed, if we represent $\mathcal{T}$ as $\sum e_{i} \otimes e^{i}$ with dual bases $\left\{e_{i}\right\}$ and $\left\{e^{i}\right\}$ in $U\left(b_{2}\right)$ and $\widetilde{F u n}$, respectively, then we obtain the relations

$$
\mathcal{T}_{1} \circ \mathcal{T}_{2}=\sum e_{i} \otimes e_{j} \otimes e^{i} \circ e^{j}=\mathcal{F}_{12}(\Delta \otimes i d)(\mathcal{T}) .
$$

Calculating $\mathcal{T}_{2} \circ \mathcal{T}_{1}$, we see that

$$
\mathcal{T}_{2} \circ \mathcal{T}_{1}=\sum e_{i} \otimes e_{j} \otimes e^{j} \circ e^{i}=\mathcal{F}_{21}\left(\Delta_{o p} \otimes i d\right)(\mathcal{T}) .
$$

Let $\mathcal{R}=\mathcal{F}_{21} \mathcal{F}^{-1}$ and $R=(\rho \otimes \rho)(\mathcal{R})$. Due to the equality $\Delta=\Delta_{\text {op }}$, we have proved the following statement. 
Theorem 3.3. $\mathcal{R} \mathcal{T}_{1} \circ \mathcal{T}_{2}=\mathcal{T}_{2} \circ \mathcal{T}_{1} ;$ consequently, $R \tilde{T}_{1} \circ \tilde{T}_{2}=\tilde{T}_{2} \circ \tilde{T}_{1}$.

This theorem allows us to construct a twist for a given Jordanian $R$-matrix (and not only for a Jordanian matrix).

Since

$$
h=\frac{1}{2} \sigma^{z}=\frac{1}{2}\left(\begin{array}{cc}
1 & 0 \\
0 & -1
\end{array}\right) \quad \text { and } \quad x=\sigma^{+}=\left(\begin{array}{cc}
0 & 1 \\
0 & 0
\end{array}\right),
$$

after simple calculations, we obtain the equality

$$
\widetilde{T}=\left(\begin{array}{cc}
e^{\varphi / 2} & e^{\varphi / 2} \cdot \omega \\
0 & e^{-\varphi / 2}
\end{array}\right) .
$$

Now it is possible to rewrite the defining relation $R \widetilde{T}_{1} \circ \widetilde{T}_{2}=\widetilde{T}_{2} \circ \widetilde{T}_{1}$ as follows:

$$
e^{\varphi / 2} \circ \omega=\omega \circ e^{\varphi / 2}+\xi e^{-\varphi / 2} .
$$

Remark 3.4. Let us recall that

$$
\mathcal{A}=\frac{\mathbb{C}\left\langle t_{11}, t_{12}\right\rangle}{R T_{1} \cdot T_{2}=T_{2} \cdot T_{1}}
$$

is a right module algebra over $\mathcal{H}_{o p}=U\left(b_{2}\right)_{o p}$. Standard considerations (note that we have to start from the algebra $\widetilde{\text { Fun }}$ which is a left $\mathcal{H}$-module and a right $\mathcal{H}_{o p}$-module) show that the algebra generated by $\widetilde{T}$ with the defining relation $R \widetilde{T}_{1} \circ \widetilde{T}_{2}=\widetilde{T}_{2} \circ \widetilde{T}_{1}$ is a right $\mathcal{H}_{o p}$-module isomorphic to $\widetilde{\text { Fun }}$ as a right $\mathcal{H}_{o p}$-module. The advantage of using $\widetilde{T}$ instead of $T$ is the existence of dual bases in the former case. The isomorphism is given by the following correspondence:

$$
\varphi^{p} \omega^{q} \longrightarrow \varphi \circ \varphi \ldots \circ \varphi \circ \omega \circ \ldots \circ \omega
$$

for brevity, we use the notation $\varphi^{p} \circ \omega^{q}$.

Let us rewrite the relation

$$
e^{\varphi / 2} \circ \omega=\omega \circ e^{\varphi / 2}+\xi e^{-\varphi / 2}
$$

as follows:

$$
\varphi \circ \omega=\omega \circ \varphi+2 \xi e^{-\varphi} .
$$

By induction, we obtain the relations

$$
e^{n \varphi} \circ \omega-\omega \circ e^{n \varphi}=2 n \xi e^{(n-1) \varphi} .
$$

Using an analytic continuation (formally, it is necessary to set $z=e^{\varphi}$ ), we see that

$$
e^{a \varphi} \circ \omega-\omega \circ e^{a \varphi}=2 a \xi e^{(a-1) \varphi} .
$$

Using induction on $m$, we get the following relations:

$$
\omega^{m} \circ e^{a \varphi}=\sum_{p=0}^{m}(-1)^{p}\left(\begin{array}{c}
m \\
p
\end{array}\right) a(a-1) \ldots(a-p+1)(2 \xi)^{p} e^{(a-p) \varphi} \circ \omega^{m-p} .
$$

Recall that to find the twist, it is necessary to compute the coefficient at 1 in the expression $\left(\varphi^{n} \circ \omega^{p}\right) \cdot\left(\varphi^{m} \circ \omega^{q}\right)$ after the reduction of this expression to the form $\sum C_{r s}^{n p, m q} \varphi^{r} \circ \omega^{s}$. In other words, it is necessary to find $C_{00}^{n p, m q}$.

From relation (3) it follows that if both $n$ and $q$ are nonzero, then $C_{00}^{q m, k n}=0$. Thus, it is necessary to compute $C_{00}^{0 m, k 0}$. In addition, it is obvious that it is enough to consider only the last term, namely,

$$
(-1)^{m}(2 \xi)^{m} a(a-1) \ldots(a-m+1) e^{(a-m) \varphi} .
$$

To find the commutation relations for the elements $\omega^{m}$ and $\varphi^{k}$, we have to differentiate relation (3) in "variable a" several times and to put $a=0$. In particular, it is easy to see that

$$
C_{00}^{0 m, k 0}=\left.\frac{(-2 \xi)^{m}}{m ! k !} \cdot \frac{d^{k}}{d a^{k}}(a)_{m}\right|_{a=0}
$$


where $(a)_{m}=a(a-1) \ldots(a-m+1)$. Thus,

$$
\mathcal{F}=\left.\sum_{m=0}^{\infty} \frac{(-1)^{m}}{m !}(2 \xi)^{m} \cdot x^{m} \otimes \sum_{k=0}^{\infty} \frac{h^{k}}{k !} \frac{d^{k}}{d a^{k}}(a)_{m}\right|_{a=0},
$$

where $h$ and $x$ are the generators of the algebra $U\left(b_{2}\right)$.

The second factor in the tensor product is the expansion of $(a)_{m}$ into the Taylor series in a neighborhood of the point $a=h$. Thus, finally we see that

$$
\mathcal{F}=\sum_{m=0}^{\infty} \frac{1}{m !}(-2 \xi x)^{m} \otimes(h)_{m}=(1 \otimes 1-2 \xi x \otimes 1)^{1 \otimes h}=\exp (\log (1-2 \xi x) \otimes h) .
$$

It is easy to check that in the fundamental representation, the Jordanian $R$-matrix is reproduced as follows:

$$
(\rho \otimes \rho)\left(\mathcal{F}_{21} \mathcal{F}^{-1}\right)=\left(\begin{array}{cccc}
1 & -\xi & \xi & \xi^{2} \\
0 & 1 & 0 & -\xi \\
0 & 0 & 1 & \xi \\
0 & 0 & 0 & 1
\end{array}\right) .
$$

\subsection{One more example of a twist for $U\left(b_{2}\right)$}

As another elementary example, we consider the following unitary quasiclassical solution of the Yang-Baxter equation (see, for example, [5]):

$$
\mathbf{R}_{\text {triang }}=\left(\begin{array}{cccc}
1 & -\xi & \xi & -\xi^{2} \\
0 & 1 & 0 & \xi \\
0 & 0 & 1 & -\xi \\
0 & 0 & 0 & 1
\end{array}\right)=1+\xi r+\mathcal{O}\left(\xi^{2}\right)
$$

where $r=\sigma^{+} \otimes \sigma^{0}-\sigma^{0} \otimes \sigma^{+}$and $\sigma^{0}=\operatorname{diag}(1,1)$. From the structure of the classical $r$-matrix it follows that to construct a carrier for the $r$-matrix, it is necessary to add the central element $c$ to the Borel algebra from the previous example. We take the following dual bases in $U$ and $U^{*}$ :

$$
h^{m} x^{k} c^{p} \quad \text { and } \quad \frac{\varphi^{m} \omega^{k} \alpha^{p}}{m ! k ! p !} .
$$

The canonical element in $U \otimes U^{*}$ is equal to

$$
\mathcal{T}=\exp (h \otimes \varphi) \exp (x \otimes \omega) \exp (c \otimes \alpha),
$$

and in the representation $\rho(c)=1, \rho(h)=\frac{1}{2} \sigma^{z}, \rho(x)=\sigma^{+}$, we have the following equality:

$$
\mathcal{T}=\left.e^{h \otimes \varphi} e^{x \otimes \omega} e^{c \otimes \alpha}\right|_{\left\{\rho: c=1, h=\frac{1}{2} \sigma^{z}, x=\sigma^{+}\right\}}=\left(\begin{array}{cc}
e^{\varphi / 2} & 0 \\
0 & e^{-\varphi / 2}
\end{array}\right)\left(\begin{array}{cc}
1 & \omega \\
0 & 1
\end{array}\right)\left(\begin{array}{cc}
e^{\alpha} & 0 \\
0 & e^{\alpha}
\end{array}\right)
$$

The relation $R T T=T T$ with $R$-matrix (4) defines the followng new (noncommutative) multiplication law for the generators $\varphi, \omega, \alpha$ of $U^{*}$ :

$$
\varphi \alpha=\alpha \varphi, \quad \varphi \omega=\omega \varphi, \quad \alpha \omega=\omega \alpha+\xi \exp (-\varphi) .
$$

To find structure constants of the new multiplication law, we take into account that the element $\varphi$ is central and the commutation relations (8) hold:

$$
\varphi^{m} \omega^{k} \alpha^{p} \circ \varphi^{m_{1}} \omega^{k_{1}} \alpha^{q}=\varphi^{m+m_{1}} \omega^{k+k_{1}} \alpha^{p+q}+\ldots .
$$

To compute the twist $\mathcal{F}$, we have to find the nonzero structure constants $m_{(000)}^{(m k p)\left(m_{1} k_{1} q\right)}$. This is possible only if we put $m=m_{1}=0, k=q=0$, and $p=k_{1}$. Using (8), we see that if $k \leq p$, then

$$
\alpha^{p} \omega^{k}=\omega^{k} \alpha^{p}+\sum_{n=0}^{k} \omega^{n} \alpha^{p-k+n} C_{k}^{n} \xi^{k-n} C_{p}^{k-n}(k-n) ! e^{-(k-n) \varphi} .
$$


Thus, the desired structure constants (with $k=p$ and $n=0$ ) are equal to

$$
m_{(000)}^{(00 p)(0 p 0)}=\frac{\xi^{p}}{p !}
$$

and the corresponding universal twist has the following form:

$$
\mathcal{F}=\sum_{p} \frac{\xi^{p}}{p !} c^{p} \otimes x^{p}=\exp (\xi c \otimes x) .
$$

In the vector representation $\mathbb{C}^{2}, \mathcal{F}$ is a $4 \times 4$-matrix and the expression $R=F_{21} F^{-1}$ coincides with the initial $R$-matrix.

\subsection{The case of the Lie superalgebra $s b_{2}$}

Consider the Lie superalgebra $s b_{2}$, which also has two generators; $h$ is even, $x$ is odd, and the commutation relations are as follows:

$$
[h, x]=x, \quad[x, x]=0 .
$$

In the universal enveloping superalgebra $U\left(s b_{2}\right)$, the commutator [,] is understood as a super-commutator $\left(\mathbb{Z}_{2^{-}}\right.$ graded commutator):

$$
[a, b]=a b-(-1)^{p(a) p(b)} b a .
$$

We take as the unitary quasiclassical solution of the $Z_{2}$-graded Yang-Baxter equation [11] the following $R$-matrix:

$$
R=\left(\begin{array}{cccc}
1 & \eta & -\eta & 0 \\
0 & 1 & 0 & -\eta \\
0 & 0 & 1 & -\eta \\
0 & 0 & 0 & 1
\end{array}\right)
$$

where $\eta$ is an odd Grassmannian parameter, $\eta^{2}=0$.

Generators of the dual algebra $U^{*}\left(s b_{2}\right):\{u, \omega:\langle u, h\rangle=1,\langle\omega, x\rangle=1\}, \omega^{2}=0$, mutually commute. The canonical element $\mathcal{T} \in U \otimes U^{*}$ is equal to

$$
\mathcal{T}=e^{h \otimes u} e^{x \otimes \omega}
$$

Similarly to the first example, the superalgebra $s b_{2}$ has the following two-dimensional representation:

$$
T=\left(\begin{array}{cc}
e^{u / 2} & -e^{u / 2} \omega \\
0 & e^{-u / 2}
\end{array}\right)
$$

We fix the following dual bases in $U$ and $U^{*}$ :

$$
h^{m} x^{k} \text { and } u^{m} \omega^{k} / m !,
$$

where $m=0,1, \cdots, k=0,1$. The relation $R T T=T T$ with $R$-matrix (12) defines a new (noncommutative) multiplication law for the generators $u$ and $\omega$ of the dual superalgebra $U^{*}$ as follows:

$$
\omega u=u \omega-2 \eta e^{-u}, \quad \omega^{2}=0
$$

To define structure constants of this new multiplication law, we take into account the commutation relations (14). To calculate $\mathcal{F}$, we have to find the structure constants $m_{(00)}^{(m k)\left(m_{1} k_{1}\right)}$ different from zero. For this purpose, it is necessary to set $m=0, k_{1}=0, m_{1}=k=1$. As a consequence of $(14)$, we obtain the relation $m_{(00)}^{(01)(10)}=-2 \eta$, and the corresponding universal twist takes the following form:

$$
\mathcal{F}=1-2 \eta x \otimes h
$$

The universal $R$-matrix,

$$
\mathcal{R}=1-2 \eta(x \otimes h-h \otimes x)=\exp (-2 \eta(x \otimes h-h \otimes x))
$$

is linear with respect to the generator $x$ due to the nilpotency of $x$ and $\eta$. 


\subsection{Twisting of the quantum superalgebra $U_{q}(g l(1 \mid 1))$}

The quantum superalgebra $U_{q}(g l(1 \mid 1))$ (see the Appendix) is an example of a case with a more complicated $R$-matrix. In the fundamental two-dimensional representation of the quantum superalgebra $U_{q}(g l(1 \mid 1))$, this $R$-matrix is equal to

$$
R^{(t)}=\left(\begin{array}{cccc}
q & 0 & 0 & \xi \\
0 & q & \omega & 0 \\
0 & 0 & q^{-1} & 0 \\
0 & 0 & 0 & q^{-1}
\end{array}\right)
$$

The relation $R^{(t)} T T=T T R$ with $R^{(t)}$-matrix (17) defines a new (noncommutative) multiplication law for the elements (generators) $\alpha, \varphi, b, g$ of $U_{q}(g l(1 \mid 1))^{*}$ occuring in the $T$-matrix. To write down explicit forms of the matrices $T_{1}$ and $T_{2}$, it is necessary to include signs related to the $Z_{2}$-grading (see the Appendix):

$$
T_{1}=T \otimes I=\left(\begin{array}{cccc}
a & 0 & u & 0 \\
0 & a & 0 & -u \\
w & 0 & d & 0 \\
0 & -w & 0 & d
\end{array}\right) \quad \text { and } \quad T_{2}=I \otimes T=\left(\begin{array}{cccc}
a & u & 0 & 0 \\
w & d & 0 & 0 \\
0 & 0 & a & u \\
0 & 0 & w & d
\end{array}\right)
$$

In the right-hand side of the $R T T=T T$-relation, we use the $R$-matrix with $\xi=0$. The only commutation relation that gives us a nonzero structure constant $m_{(0)}^{(j)(k)}$ with nonzero multiindices needed to calculate the twist $\mathcal{F}$ is

$$
u^{2}=\frac{\xi}{q+q^{-1}} d^{2}, \quad \text { or } \quad b^{2}=\frac{\xi}{q+q^{-1}} 1+\cdots,
$$

where terms containing generators different from the unit are omitted. Thus, $m_{(0000)}^{(0001)(0001)}=-\xi /\left(q+q^{-1}\right)$, and the twist element is

$$
\mathcal{F}=\exp \left(-\frac{\xi}{q+q^{-1}} e \otimes e\right)=1-\frac{\xi}{q+q^{-1}} e \otimes e .
$$

\section{Discussion}

In a similar way, it is possible to consider the quantum group $U_{q}\left(g \ell_{n}\right)$. It is known that the dual Hopf algebra $\operatorname{Fun}_{q}\left(G L_{n}\right)$ which is the algebra of functions on the quantum group can be obtained from the relation $R_{q} T_{1} T_{2}=$ $T_{2} T_{1} R_{q}$, where $R_{q}$ is the Drinfel'd-Jimbo $R$-matrix . The defined algebra denoted by $R_{q}^{\prime} T_{1} T_{2}=T_{2} T_{1} R_{q}$ is a right module algebra over $U_{q}\left(g \ell_{n}\right)_{o p}$. Here $R_{q}^{\prime}$ is another $R$-matrix such that $R_{q}^{\prime} \mathcal{P}$ and $\mathcal{P} R_{q}$ have the same spectrum $\left(\mathcal{P}\right.$ is the permutation operator in $\left.\mathbb{C}^{n} \otimes \mathbb{C}^{n}\right)$. In this case, there exists a universal twist $\mathcal{F} \in U_{q}\left(g \ell_{n}\right)^{\otimes 2}$ such that $R_{q}^{\prime}=(\rho \otimes \rho)\left(\mathcal{F}_{21} \mathcal{R}_{q} \mathcal{F}^{-1}\right)$, where $\mathcal{R}_{q}$ is the universal $R$-matrix and $\rho: U_{q}\left(g \ell_{n}\right) \rightarrow \operatorname{End}\left(\mathbb{C}^{n}\right)$ is the fundamental vector representation of the Hopf algebra $U_{q}\left(g \ell_{n}\right)$.

\section{APPENDIX}

\section{A. Universal $R$-matrix for $g l_{q}(1 \mid 1)$}

The superalgebra $U_{q}(g l(1 \mid 1))$ is generated by two even elements $h$ and $c$ and by two odd elements $e$ and $f$, where $c$ is central, and the remaining generators satisfy the following relations:

$$
[h, e]=2 e, \quad[h, f]=-2 f, \quad e^{2}=f^{2}=0, \quad \text { and } \quad[e, f]_{+}=\left(q^{c}-q^{-c}\right) /\left(q-q^{-1}\right) .
$$

The $Z_{2}$-graded structure of the Hopf algebra is defined by the following coproducts:

$$
\begin{aligned}
& \Delta(h)=h \otimes 1+1 \otimes h, \\
& \Delta(c)=c \otimes 1+1 \otimes c, \\
& \Delta(e)=e \otimes q^{c}+1 \otimes e,
\end{aligned}
$$

and

$$
\Delta(f)=f \otimes 1+q^{-c} \otimes f,
$$

and the counit $\varepsilon(h)=\varepsilon(c)=\varepsilon(e)=\varepsilon(f)=0$. The algebra $U_{q}(g l(1 \mid 1))$ is quasitriangular with the universal $R$-matrix,

$$
\mathcal{R}=q^{\frac{1}{2}(c \otimes h+h \otimes c)}(1-\omega e \otimes f) \in U_{q}(g l(1 \mid 1))^{\otimes 2},
$$


where $\omega=\left(q-q^{-1}\right)$. This universal $R$-matrix intertwines the coproduct with the opposite coproduct.

Let us note that the superalgebra $g l_{q}(1 \mid 1)$ admits an Abelian twist since it has a two-dimensional classical Abelian subalgebra spanned by the elements $c$ and $h$ with a primitive coproduct. There is a special choice of a twist element with the following property: one of odd generators, say $e$, becomes a primitive element. Indeed,

$$
\Delta_{t}(e)=e^{\alpha h \otimes c} \Delta(e) e^{-\alpha h \otimes c}=e \otimes q^{c} e^{2 \alpha c}+1 \otimes e=e \otimes 1+1 \otimes e
$$

if we set $\alpha=-\frac{1}{2} \log (q)$.

Hence, there exists another twist $\mathcal{T}_{1}=\exp (\xi e \otimes e)$. As a result of twisting by this element, we obtain a new universal $R$-matrix:

$$
\widetilde{\mathcal{R}}=\exp (-\xi e \otimes e) e^{\alpha c \otimes h} \mathcal{R} e^{-\alpha h \otimes c} \exp (-\xi e \otimes e)
$$

having an off-diagonal parameter in the matrix representation.

B. Dual Hopf algebra $U_{q}(g l(1 \mid 1))^{*}$

Generators of the coquasitriangular Hopf algebra dual to $U_{q}(g l(1 \mid 1))$ are defined by the following nonzero pairings:

$$
\langle\varphi, h\rangle=1, \quad\langle\alpha, c\rangle=1, \quad\langle b, e\rangle=1, \quad \text { and } \quad\langle g, f\rangle=1 .
$$

The generators $\varphi$ and $\alpha$ are even, the generators $b$ and $g$ are odd. The canonical element

$$
\mathcal{T} \in U_{q}(g l(1 \mid 1)) \otimes U_{q}(g l(1 \mid 1))^{*}
$$

is defined by the standard exponentials due to the nilpotency of the generators $e$ and $f$ (and $b$ and $g$ ) as follows:

$$
\mathcal{T}=\exp (f \otimes g) \exp (c \otimes \alpha) \exp (h \otimes \varphi) \exp (e \otimes b)
$$

and

$$
\mathcal{T}=(1+f \otimes g) \exp (c \otimes \alpha) \exp (h \otimes \varphi)(1+e \otimes b)
$$

The duality relations allow us to calculate the coproduct and to find the multiplication law for the generators of the dual Hopf algebra:

$$
\begin{gathered}
\Delta_{*}(\varphi)=\varphi \otimes 1+1 \otimes \varphi, \\
\Delta_{*}(\alpha)=\alpha \otimes 1+1 \otimes \alpha-\frac{2 \eta}{\omega} b \otimes g, \\
\Delta_{*}(b)=b \otimes \exp (-2 \varphi)+1 \otimes b, \\
\Delta_{*}(g)=g \otimes 1+\exp (-2 \varphi) \otimes g, \\
{[\alpha, b]=-\eta b, \quad[\alpha, g]=-\eta g, \quad b^{2}=g^{2}=0, \quad[b, g]_{+}=0 .}
\end{gathered}
$$

To check that the canonical element $\mathcal{T}$ is a bicharacter, i.e.,

$$
(\Delta \otimes 1) \mathcal{T}=\mathcal{T}_{13} \mathcal{T}_{23} \quad \text { and } \quad\left(1 \otimes \Delta_{*}\right) \mathcal{T}=\mathcal{T}_{12} \mathcal{T}_{13}
$$

it is necessary (using the coproduct $\Delta$ in the Hopf algebra $U_{q}(g l(1 \mid 1))$ and the dual coproduct $\Delta_{*}$ in $\left.U_{q}(g l(1 \mid 1))^{*}\right)$ to take into account a particular form of the Baker-Campbell-Hausdorff formula,

$$
\exp (t X+Y)=\exp (t X) \exp \left(\frac{1-e^{-t}}{t} Y\right), \quad[X, Y]=Y
$$

This formula allows us to extract a factor in $\left(1 \otimes \Delta_{*}\right) \mathcal{T}$, namely,

$$
\left.\exp \left(c \otimes\left(\alpha \otimes 1+1 \otimes \alpha-\frac{2 \eta}{\omega} b \otimes g\right)\right)=\exp (c \otimes \alpha \otimes 1) \exp \left(-\frac{\left(q^{c}-q^{-c}\right)}{\omega} b \otimes g\right)\right) \exp (c \otimes 1 \otimes \alpha)
$$

due to the commutation relation

$$
[c \otimes \alpha \otimes 1+c \otimes 1 \otimes \alpha, 1 \otimes b \otimes g]=-2 \eta c \otimes b \otimes g .
$$


In the two-dimensional representation of the Hopf superalgebra $U_{q}(g l(1 \mid 1))$, elements of the $2 \times 2$-matrix $T=$ $\left(\begin{array}{ll}a & u \\ w & d\end{array}\right)$ can be expressed in terms of generators of the dual superalgebra $U_{q}(g l(1 \mid 1))^{*}$ as follows:

$$
a=e^{(\alpha+\varphi)}, \quad u=e^{(\alpha+\varphi)} b, \quad w=g e^{(\alpha+\varphi)}, \quad d=e^{(\alpha-\varphi)}+g e^{(\alpha+\varphi)} b .
$$

This research was supported by the Russian Foundation for Basic Research, grants 00-01-00500 and 02-0100085.

Translated by A. A. Stolin, P. P. Kulish, and E. V. Damaskinsky.

\section{REFERENCES}

1. V. Drinfel'd, "Quantum groups," in: Proc. Intern. Congr. Math., Berkeley 1986. AMS, Providence, RI, 1987, p. 798.

2. V. Drinfel'd, "Constant semiclassical solutions of the quantum Yang-Baxter equation," Dokl. Akad. Nauk SSSR, 273, 531-535 (1983).

3. V. Drinfel'd, "Quasi-Hopfian algebras," Algebra Analiz, 114-148 (1989).

4. N. Yu. Reshetikhin, L. A. Takhtajan, and L. D. Faddeev, "Quantization of Lie groups and Lie algebras," Algebra Analiz, 1, 178-206 (1989).

5. P. P. Kulish, V. D. Lyakhovsky, and A. I. Mudrov, "Extended Jordanian twists for Lie algebras," J.Math. Phys., 40, 4569-4581 (1999).

6. V. D. Lyakhovsky, A. A. Stolin, and P. P. Kulish, "Chains of Frobenius subalgebras of $s o(M)$ and the corresponding twists," J. Math. Phys., 42, 5006-5019 (2001).

7. S. Parmentier, "On coproducts of quasitriangular Hopf algebras," Algebra Analiz, 6, 204-222 (1994).

8. S. Zakrzewski, "A characterization of coboundary Poisson-Lie groups and Hopf algebras," Banach Center Publ., 40, 273-278 (1997).

9. E. E. Demidov, Yu. I. Manin, E. E. Mukhin, and D. V. Zhdanovich, "Nonstandard quantum deformations of $G L(n)$ and constant solutions of the Yang-Baxter equation," in: Yukawa Seminar Common Trends in Mathematics and Quantum Field Theories, T. Eguchi, T. Inami, and T.Miwa (eds.), Prog. Theor. Phys. Suppl., 102 (1990), pp. 203-218.

10. P. P. Kulish and A. I. Mudrov, "Quantization of inhomogeneous Lie bialgebras," J.Geom. Phys., 42, 64-77 (2002).

11. P. P. Kulish and E. K. Sklyanin, "Quantum spectral transform method: recent developments," in: Integrable Quantum Field Theories, J. Hietarinta and C. Montonen (eds.), Lect. Notes Phys., 151 (1982), pp. 61-117. 